\title{
Effect of Ligands Coordinated at Platinum Atom on Redox Properties of Binuclear Manganese-Platinum Phenylvinylidene Complexes
}

\author{
Galina V. Burmakina*a,b, \\ Victor V. Verpekin a, Dmitry V. Zimonina, \\ Oleg S. Chudin a and Anatoly I. Rubaylo ${ }^{\text {a,b }}$ \\ anstitute of Chemistry and Chemical Technology SB RAS \\ FRC "Krasnoyarsk Scientific Center of the SB RAS" \\ 50/24 Akademgorodok, Krasnoyarsk, 660036, Russia \\ 'Siberian Federal University \\ 79 Svobodny, Krasnoyarsk, 660041, Russia
}

Received 11.09.2018, received in revised form 25.10.2018, accepted 28.11.2018

The redox properties of binuclear heterometallic complexes $C p(C O)_{2} \operatorname{MnPt}\left(\mu-C^{l}=C^{2} H P h\right)(L)\left(L^{\prime}\right)$ $\left[L=L^{\prime}=P P h_{3}(1) ; L=P P h_{2} H ; L^{\prime}=P P h_{3}(2) ; L=C O, L^{\prime}=P P h_{3}(3) ; L=L^{\prime}=P\left(O P r^{i}\right)_{3}(4) ; L=P P h_{2} H\right.$; $\left.L^{\prime}=P\left(\mathrm{OPr}^{i}\right)_{3}(5) ; L=C O, L^{\prime}=P\left(\mathrm{OPr}^{i}\right)_{3}(\mathbf{6})\right]$ were studied by electrochemical methods at platinum, glassed carbon and dropping mercury electrodes in acetonitrile. Electrochemical behavior of the complexes were shown to depend on the nature of L, L' ligands at the platinum atom. It was established that one-electron oxidation of 1, 2, 4, 5 results in a cleavage of the Mn-Pt and the Pt-Cl bonds to give mononuclear phenylvinylidene complex $\mathrm{Cp}(\mathrm{CO})_{2} \mathrm{Mn}=\mathrm{C}=\mathrm{CHPh}$ (7) and [PtLL'] fragments. The quasireversible one-electron oxidation of $\mathbf{3}$ and $\mathbf{6}$ is indicating their stronger electrochemical stability in redox-reaction compare to $1,2,4,5$.

Keywords: electrochemistry, redox properties, binuclear complexes, phenylinylidene, manganese, platinum, phosphorus containing ligands.

Citation: Burmakina G.V., Verpekin V.V., Zimonin D.V., Chudin O.S., Rubaylo A.I. Effect of ligands coordinated at platinum atom on redox properties of binuclear manganese-platinum phenylvinylidene complexes, J. Sib. Fed. Univ. Chem., 2018, 11(4), 543-551. DOI: 10.17516/1998-2836-0098.

(C) Siberian Federal University. All rights reserved

* Corresponding author E-mail address: bgvicct@gmail.com,bgv@akadem.ru 


\title{
Влияние лигандного окружения атома платины на редокс-свойства биядерных марганец-платиновых фенилвинилиденовых комплексов
}

\author{
Г.В. Бурмакина ${ }^{\mathrm{a}, \tilde{0}}$, В.В. Верпекин ${ }^{\mathrm{a}}$, \\ Д.В. Зимонин ${ }^{\mathrm{a}}$, О.С. Чудин ${ }^{\mathrm{a}}$, А.И. Рубайло ${ }^{\mathrm{a}, \overline{0}}$ \\ ${ }^{a}$ Институт химии и химической технологии СО РАН \\ ФИЦ «Красноярский научный иентр СО РАН» \\ Россия, 660036, Красноярск, Академгородок,50/24 \\ ${ }^{6}$ Сибирский федеральный университет \\ Россия, 660041, Красноярск, пр. Свободный, 79
}

Редокс-свойства биядерных гетерометаллических комплексов: $C p(C O)_{2} \operatorname{MnPt}\left(\mu-C^{l}=C^{2} H P h\right)$ $(L)\left(L^{\prime}\right)\left[L=L^{\prime}=P P h_{3}(\mathbf{1}) ; L=P P h_{2} H ; L^{\prime}=P P h_{3}(2) ; L=C O, L^{\prime}=P P h_{3}(3) ; L=L^{\prime}=P\left(O P r^{i}\right)_{3}(4)\right.$; $\left.L=P P h_{2} H ; L^{\prime}=P\left(O P r^{i}\right)_{3}(5) ; L=C O, L^{\prime}=P\left(O P r^{i}\right)_{3}(6)\right]$, изучены электрохимическими методами на платиновом, стеклоуглеродном и ртутном капельном электродах в ацетонитриле. Показано, что значения редокс-потенциалов комплексов и их электрохимическое поведение зависят от природы лигандов L, L' при атоме платины. Установлено, что одноэлектронное окисление комплексов 1, 2, 4, 5 приводит к разрыву связей Mn-Pt, Pt-Cl, образованию моноядерного фенилвинилиденового комплекса марганца $\mathrm{Cp}(\mathrm{CO})_{2} \mathrm{Mn}=\mathrm{C}=\mathrm{CHPh}$ (7) $u$ [PtLL'] фрагментов. Одноэлектронное окисление комплексов 3, 6 протекает квазиобратимо, что свидетельствует об их большей устойчивости в редокс-реакииях по сравнению с 1, 2, 4, 5 .

Ключевые слова: электрохимия, редокс-свойства, биядерные комплексы, фенилвинилиден, марганеи, платина, фосфорсодержащие лиганды.

\section{Введение}

Моно- и полиядерные соединения переходных металлов, в том числе платины и марганца, перспективны в качестве моделей каталитических систем в реакциях превращения органических молекул на металлических центрах [1-12]. Особый интерес представляют гетерометаллические комплексы благодаря тому, что взаимодействие металл - реагент на гетерометаллических катализаторах может происходить между несколькими атомами органического субстрата и различными атомами металлов, увеличивая разнообразие в способах координации, и может принципиально изменить реакционную способность и физико-химические свойства субстрата [11-13]. Одними из таких соединений являются гетерометаллические комплексы и кластеры, стабилизированные мостиковыми винилиденовыми лигандами [14-16].

Электронное состояние металлического центра в металлокомплексах является одним из основных параметров, определяющих их химические и физико-химические свойства. Изменение этого параметра может быть осуществлено модификацией лигандного окружения одного из атомов металла, что позволяет целенаправленно менять свойства комплекса, в том числе 
и его способность участвовать в различных реакциях с определенными реагентами [10-16]. Основными методами, позволяющими изучать влияние лигандного окружения на изменение электронного состояния комплексов в редокс-реакциях, являются электрохимические методы [17-20].

Настоящая работа посвящена электрохимическому изучению редокс-свойств биядерных марганец-платиновых $\mu$-фенилвинилиденовых комплексов 1-6, в которых лиганды L и L' при атоме платины различаются по своим электронным свойствам ( $\sigma$-донорной и $\pi$-акцепторной способностям) и стерическим параметрам (коническими углами, $\theta$ ), и установлению закономерностей их изменения в зависимости от природы лигандного окружения атома платины:

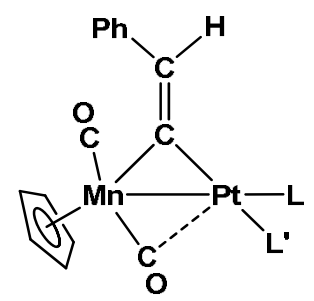

$$
\begin{aligned}
& \mathrm{L}=\mathrm{L}^{\prime}=\mathrm{PPh}_{3}(\mathbf{1}) \\
& \mathrm{L}=\mathrm{PPh}_{3}, \mathrm{~L}^{\prime}=\mathrm{PPh}_{2} \mathrm{H}(\mathbf{2}) \\
& \mathrm{L}=\mathrm{PPh}_{3}, \mathrm{~L}^{\prime}=\mathrm{CO}(\mathbf{3}) \\
& \mathrm{L}=\mathrm{L}^{\prime}=\mathrm{P}\left(\mathrm{OPr}^{\prime}\right)_{3} \text { (4) } \\
& \mathrm{L}=\mathrm{P}\left(\mathrm{OPr}^{\mathrm{i}}\right)_{3}, \mathrm{~L}^{\prime}=\mathrm{PPh}_{2} \mathrm{H}(\mathbf{5}) \\
& \mathrm{L}=\mathrm{P}\left(\mathrm{OPr}^{\prime}\right)_{3}, \mathrm{~L}^{\prime}=\mathbf{C O}(\mathbf{6})
\end{aligned}
$$

Соединения 1-6 исследованы методами полярографии, циклической вольтамперометрии (ЦВА) и электролиза при контролируемом потенциале (ЭКП) в ацетонитриле. Следует отметить, что ранее нами было изучено только полярографическое восстановление комплексов $\mathbf{1 , 3}$, 4, 6 на р.к.э. [21, 22], в данной работе приведены результаты исследования процессов как восстановления, так и окисления этих соединений, комплексы 2,5 изучены впервые.

\section{Экспериментальная часть}

Комплексы $\mathbf{1}, \mathbf{3}, \mathbf{4 ,} 6$ и $\mathbf{2}, \mathbf{5}$ синтезированы по методикам [23, 24] и [25] соответственно. Электрохимические измерения проводили в свежеприготовленных растворах соединений в ацетонитриле, очищенном по методике [26], с добавлением $0.1 \mathrm{M} \mathrm{Et}_{4} \mathrm{NBF}_{4}$ в качестве фонового электролита, в атмосфере аргона при комнатной температуре. Полярограммы и циклические вольтамперограммы (ЦВА) регистрировали на потенциостате IPC-Pro М (ООО НТФ «Вольта», Санкт-Петербург, Россия). В качестве рабочих электродов ${ }^{1}$ использовали ртутный капельный электрод (р.к.э.) с принудительным отрывом капель $(\mathrm{m}=3.6 \mathrm{мг} / \mathrm{c}, \tau=0.23$ с) и стационарные: платиновый $(\mathrm{Pt})(\mathrm{d}=1$ мм) или стеклоуглеродный $(\mathrm{CV})(\mathrm{d}=5$ мм) электроды, в тефлоновых корпусах (d = 10 мм). Электродом сравнения служил полуэлемент $\mathrm{Ag} / 0.1 \mathrm{M} \mathrm{AgNO}_{3}$ в $\mathrm{MeCN}$, coединенный с ячейкой электролитическим мостом, заполненным фоновым электролитом, через капилляр Луггина. В качестве вспомогательного электрода применяли платиновую спираль, помещенную в стеклянную трубку с пористым фильтром. Число электронов, участвующих в каждой редокс-стадии, определяли сравнением высот волн исследуемых соединений с высотой одноэлектронной волны хорошо изученного комплекса $\mathrm{Cp}(\mathrm{CO})_{2} \mathrm{Mn}=\mathrm{C}=\mathrm{CHPh}(7)[21,22]$. Электролиз при контролируемом потенциале (ЭКП) проводили на потенциостате IPC-Pro M (рабочий электрод - платиновая пластина площадью 4 см²$^{2}$ электрод сравнения $-\mathrm{Ag} / 0.1 \mathrm{M}$

\footnotetext{
Использование различных рабочих электродов позволяет исследовать процессы окисления и восстановления соединений в более широкой области потенциалов. Так, рабочая область измерения потенциалов в ацетонитриле (относительно $\mathrm{Ag} / 0.1 \mathrm{M} \mathrm{AgNO}_{3}$ в $\mathrm{MeCN}$ ) на р.к.э. находится в пределах от 0.30 до -3.20 B, на Pt- и СУ-электродах от 2.0 до -2.2 и от 2.0 до -2.6 В соответственно.
}

$$
-545-
$$


$\mathrm{AgNO}_{3}$ в $\mathrm{MeCN}$, вспомогательный - $\mathrm{Pt}$ ) при интенсивном барботировании аргоном. Химическое окисление комплексов 1,2 и 4,5 проводили с использованием борфторида ферроцения [Fc] $\left[\mathrm{BF}_{4}\right]\left(\mathrm{Fc}=\left[\left(\mathrm{C}_{5} \mathrm{H}_{5}\right)_{2} \mathrm{Fe}\right]^{+}\right)[27]$.

Регистрацию ИК-спектров осуществляли на ИК-Фурье спектрометре Vector 22 (фирмы Bruker) в области валентных колебаний СО-групп $\left(1600-2200 \mathrm{~cm}^{-1}\right)$ в кюветах с толщиной слоя 0.646 мм. Кюветы предварительно продували аргоном.

\section{Результаты и обсуждение}

Полученные электрохимические характеристики фенилвинилиденовых биядерных марганец-платиновых комплексов 1-6 и исходного моноядерного комплекса марганца $\mathrm{Cp}(\mathrm{CO})_{2} \mathrm{Mn}=\mathrm{C}=\mathrm{CHPh}(7)$ приведены в табл., циклические вольтамперограммы комплексов 1-3 и 7 на СУ электроде - на рис. 1.

Как видно из данных табл., комплексы 1-6 способны как восстанавливаться на р.к.э., так и окисляться на Pt и СУ электродах. Значения потенциалов первых волн окисления биядерных комплексов 1 и $\mathbf{4}$, содержащих два $\mathrm{PPh}_{3}$ или $\mathrm{P}\left(\mathrm{OPr}^{\mathrm{i}}\right)_{3}$ лиганда при атоме платины, находятся в отрицательной области потенциалов, при этом величины $\mathrm{E}_{1 / 2}$ окисления комплекса 4 смещены в катодную область по сравнению с 1.

При замещении одного из фосфорсодержащих лигандов $\mathrm{PPh}_{3}$ или $\mathrm{P}\left(\mathrm{OPr}^{\mathrm{i}}\right)_{3}$ при атоме платины в комплексах 1 и 4 на другие лиганды, отличающиеся по электронодонорной способности и размеру, наблюдаются две тенденции в зависимости от природы вводимого лиганда:

1. Замещение одного из б-донорных фосфорсодержащих лигандов в 1 и 4 на $\pi$-акцепторный карбонильный лиганд приводит к значительному смещению значений первых потенциалов окисления комплексов 3 и 6 в анодную область, настолько, что комплексы 3 и 6 окисляются в положительной области потенциалов. При этом для комплекса $\mathbf{6}$, содержащего более слабый б-донорный лиганд $\mathrm{P}\left(\mathrm{OPr}^{\mathrm{i}}\right)_{3}$, это смещение выражено сильнее (табл.).

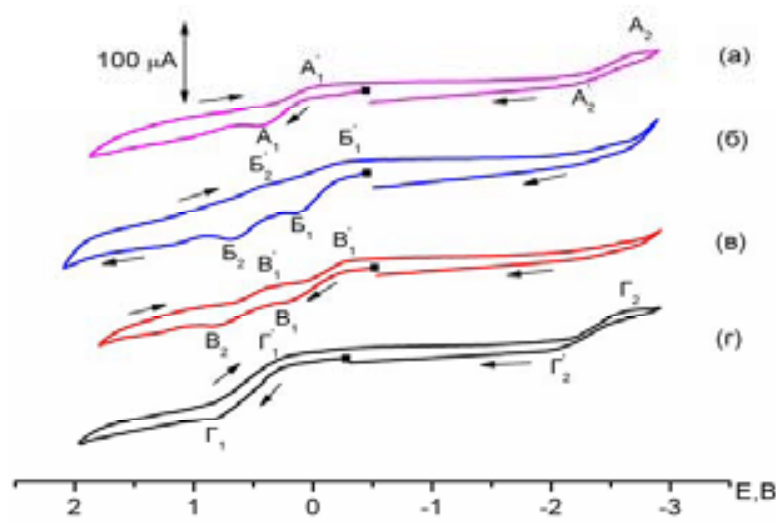

Рис. 1. Циклические вольтамперограммы комплексов: a $-\mathrm{Cp}(\mathrm{CO}){ }_{2} \mathrm{MnPt}(\mu-\mathrm{C}=\mathrm{CHPh})(\mathrm{CO})\left(\mathrm{PPh}_{3}\right)(3) ; \sigma-$ $\mathrm{Cp}(\mathrm{CO})_{2} \mathrm{MnPt}(\mu-\mathrm{C}=\mathrm{CHPh})\left(\mathrm{PPh}_{3}\right)_{2}(\mathbf{1}) ; \mathrm{B}-\mathrm{Cp}(\mathrm{CO})_{2} \mathrm{MnPt}(\mu-\mathrm{C}=\mathrm{CHPh})\left(\mathrm{PPh}_{3}\right)\left(\mathrm{PPh}_{2} \mathrm{H}\right)(\mathbf{2}) ; \Gamma-\mathrm{Cp}(\mathrm{CO})_{2} \mathrm{Mn}=\mathrm{C}=\mathrm{CHPh}$ (7) $\left(\mathrm{CV}, \mathrm{MeCN}, 0.1 \mathrm{M} \mathrm{Et}_{4} \mathrm{NBF}_{4}, \mathrm{Ag} / 0.1 \mathrm{M} \mathrm{AgNO}_{3}\right.$ в $\left.\mathrm{MeCN}, \mathrm{C}=1 \mathrm{MM}, \mathrm{V}=25 \mathrm{MB} / \mathrm{c}\right)$

Fig. 1. Cyclic voltammograms of complexes: a $-\mathrm{Cp}(\mathrm{CO})_{2} \mathrm{MnPt}(\mu-\mathrm{C}=\mathrm{CHPh})(\mathrm{CO})\left(\mathrm{PPh}_{3}\right)(\mathbf{3}) ; \sigma-\mathrm{Cp}(\mathrm{CO})_{2} \mathrm{MnPt}(\mu-$ $\mathrm{C}=\mathrm{CHPh})\left(\mathrm{PPh}_{3}\right)_{2}(\mathbf{1})$; в $-\mathrm{Cp}(\mathrm{CO})_{2} \mathrm{MnPt}(\mu-\mathrm{C}=\mathrm{CHPh})\left(\mathrm{PPh}_{3}\right)\left(\mathrm{PPh}_{2} \mathrm{H}\right)(\mathbf{2}) ; \quad$ - $-\mathrm{Cp}(\mathrm{CO})_{2} \mathrm{Mn}=\mathrm{C}=\mathrm{CHPh}$ (7) (GC, $\mathrm{MeCN}, 0.1 \mathrm{M} \mathrm{Et}_{4} \mathrm{NBF}_{4}, \mathrm{Ag} / 0.1 \mathrm{M} \mathrm{AgNO}_{3}$ in $\left.\mathrm{MeCN}, \mathrm{C}=1 \mathrm{mM}, \mathrm{V}=25 \mathrm{mV} / \mathrm{s}\right)$ 
2. При замещении одного из лигандов $\mathrm{PPh}_{3}$ или $\mathrm{P}\left(\mathrm{OPr}^{\mathrm{i}}\right)_{3}$ в 1 и 4 на дифенилфосфиновый происходит небольшое смещение значений $\mathrm{E}_{1 / 2}$ первых волн окисления комплексов 2 и 5 соответственно, в катодную область потенциалов (табл.).

Следует отметить, что комплексы с трифенилфосфиновыми лигандами 1-3 восстанавливаются при более положительных значениях потенциалов по сравнению с изопропилфосфитными комплексами 4-6. Аналогичные тенденции влияния природы фосфорсодержащих лигандов $\left[\mathrm{PPh}_{3}, \mathrm{PPh}_{2} \mathrm{H}, \mathrm{P}\left(\mathrm{OPr}^{\prime}\right)_{3}\right]$ наблюдаются и при восстановлении комплексов 1-6 (табл.).

Таким образом, полученные результаты показывают, что значения редокс-потенциалов биядерных MnPt комплексов 1-6 зависят от природы лигандов L, L' при атоме платины. Известно [28], что изменения структуры и свойств моноядерных комплексов типа $\mathrm{Ni}(\mathrm{CO})_{\mathrm{n}-1}\left(\mathrm{PR}_{3}\right)$ $[\mathrm{R}=\mathrm{H}$, alkyl, aryl $]$ обусловлены такими параметрами лигандов $\mathrm{PR}_{3}$, как их электронодонорная способность и размер (стерический эффект $S_{\text {eff }}$ - обусловлен размером $\mathrm{PR}_{3}$ лиганда и приближенно оценивается величиной конического угла $\boldsymbol{\theta}$ ). Согласно теоретическим расчетам и экспериментальным данным [28, 29], электронодонорная способность фосфорсодержащих лигандов увеличивается в ряду: $\mathrm{P}\left(\mathrm{OPr}^{\prime}\right)_{3}<\mathrm{PPh}_{2} \mathrm{H}<\mathrm{PPh}_{3}$, а их размер (конический угол, $\theta$ ) в следующем порядке: $\mathrm{PPh}_{2} \mathrm{H}<\mathrm{P}\left(\mathrm{OPr}^{i}\right)_{3}<\mathrm{PPh}_{3}$. Поэтому при замене двух лигандов $\mathrm{PPh}_{3}$ в комплексе 1 на $\mathrm{P}\left(\mathrm{OPr}^{\mathrm{i}}\right)_{3}$ лиганды или одного из $\mathrm{PPh}_{3}$ в 1 на $\mathrm{PPh}_{2} \mathrm{H}$ лиганд следовало бы ожидать смещения редокс-потенциалов комплексов 4 и 2 в анодную область по сравнению с 1. Однако значения

Таблица. Электрохимические характеристики моно- и биядерных фенилвинилиденовых комплексов марганца (MeCN, $0.1 \mathrm{M} \mathrm{Et}_{4} \mathrm{NBF}_{4}, 1 \mathrm{mM}, \mathrm{Ag} / 0.1 \mathrm{M} \mathrm{AgNO}_{3}$ в $\mathrm{MeCN}$ )

Table. Electrochemical properties of mono- and binuclear phenylinylidene manganese complexes (MeCN, $0.1 \mathrm{M}$ $\mathrm{Et}_{4} \mathrm{NBF}_{4}, 1 \mathrm{mM}, \mathrm{Ag} / 0.1 \mathrm{M} \mathrm{AgNO}_{3}$ in $\mathrm{MeCN}$ )

\begin{tabular}{|c|c|c|c|}
\hline \multirow{3}{*}{ Соединение } & \multicolumn{3}{|c|}{$\mathrm{E}_{1 / 2}, \mathrm{~B}(\mathrm{n})$} \\
\hline & \multicolumn{2}{|c|}{ Окисление } & \multirow{2}{*}{$\begin{array}{c}\text { Восстановление } \\
\text { Р.к.э. }\end{array}$} \\
\hline & $\mathrm{Pt}$ & CY & \\
\hline $\mathrm{Cp}(\mathrm{CO})_{2} \operatorname{MnPt}(\mu-\mathrm{C}=\mathrm{CHPh})\left(\mathrm{PPh}_{3}\right)_{2}(\mathbf{1})$ & $\begin{array}{c}-0.09(1) \\
0.23(<1) \\
0.32(1)\end{array}$ & $\begin{array}{l}-0.05(1) \\
0.34(<1)\end{array}$ & $\begin{array}{l}-2.37(1) \\
-2.69(1) \\
-2.91(1)\end{array}$ \\
\hline $\mathrm{Cp}(\mathrm{CO})_{2} \mathrm{MnPt}(\mu-\mathrm{C}=\mathrm{CHPh})\left(\mathrm{PPh}_{3}\right)\left(\mathrm{PPh}_{2} \mathrm{H}\right)(\mathbf{2})$ & $\begin{array}{c}-0.11(1) \\
0.26(<1) \\
0.33(1)\end{array}$ & $\begin{array}{c}-0.10(1) \\
0.36(1)\end{array}$ & $-2.55(1)$ \\
\hline $\mathrm{Cp}(\mathrm{CO})_{2} \operatorname{MnPt}(\mu-\mathrm{C}=\mathrm{CHPh})(\mathrm{CO})\left(\mathrm{PPh}_{3}\right)(\mathbf{3})$ & $0.15^{\mathrm{a}}(1)$ & $0.16^{\mathrm{a}}(1)$ & $\begin{array}{l}-2.10(1) \\
-2.68(1)\end{array}$ \\
\hline $\mathrm{Cp}(\mathrm{CO})_{2} \mathrm{MnPt}(\mu-\mathrm{C}=\mathrm{CHPh})\left[\mathrm{P}\left(\mathrm{OPr}^{\mathrm{i}}\right)_{3}\right]_{2}$ (4) & $\begin{array}{c}-0.14(1) \\
0.34(1)\end{array}$ & $\begin{array}{c}-0.10(1) \\
0.36(1)\end{array}$ & $-2.60(1)$ \\
\hline $\mathrm{Cp}(\mathrm{CO})_{2} \mathrm{MnPt}(\mu-\mathrm{C}=\mathrm{CHPh})\left[\mathrm{P}\left(\mathrm{OPr}_{3}\right)_{3}\right]\left(\mathrm{PPh}_{2} \mathrm{H}\right)(\mathbf{5})$ & $\begin{array}{l}-0.15(1) \\
0.13(<1) \\
0.30(<1)\end{array}$ & $\begin{array}{l}-0.12(1) \\
0.36(<1)\end{array}$ & $-2.66(1)$ \\
\hline $\mathrm{Cp}(\mathrm{CO})_{2} \operatorname{MnPt}(\mu-\mathrm{C}=\mathrm{CHPh})(\mathrm{CO})\left[\mathrm{P}\left(\mathrm{OPr}^{\mathrm{i}}\right){ }_{3}\right](\mathbf{6})$ & $0.19^{\mathrm{a}}(1)$ & $0.22^{\mathrm{a}}(1)$ & $\begin{array}{l}-2.23(1) \\
-2.68(1) \\
-2.86(1)\end{array}$ \\
\hline $\mathrm{Cp}(\mathrm{CO})_{2} \mathrm{Mn}=\mathrm{C}=\mathrm{CHPh}(7)$ & $0.30(1)$ & $0.34(1)$ & $-1.99^{\mathrm{a}}(1)$ \\
\hline
\end{tabular}

Примечание: $\mathrm{n}$ - число электронов, участвующих в каждой электрохимической стадии (знак «<» означает, что высота волны исследуемого соединения меньше, чем высота одноэлектронной волны); а - квазиобратимая стадия.

$$
-547-
$$


$\mathrm{E}_{1 / 2}$ первых волн окисления и восстановления комплексов 4 и 2 смещаются в катодную область потенциалов по сравнению с 1. Таким образом, для трифенилфосфиновых комплексов наблюдается необычная зависимость значений редокс-потенциалов от электронодонорных свойств фосфорсодержащих лигандов при атоме платины. Причиной такого поведения, как показано в работе [30] для аналогичных комплексов, является перенос избыточной электронной плотности с атома платины на $\pi^{*}$-орбитали одного из карбонильных лигандов при соседнем атоме марганца, результатом которого является образование полумостикового взаимодействия $\mathrm{CO}_{\text {пм }}$..Pt. При этом степень такого взаимодействия зависит в большей степени от стерического размера фосфорсодержащего лиганда при атоме платины, чем от его электронодонорной способности [30]. Так, меньший конический угол $\theta$ дифенилфосфинового лиганда $\left(128^{\circ}\right)$ по сравнению с трифенилфосфиновым $\left(\theta=145^{\circ}\right)$ в комплексе 2 приводит к усилению полумостикового взаимодействия $\mathrm{CO}_{\text {пм }} \leftarrow \mathrm{Pt}$, перераспределению электронной плотности в платиносодержащем фрагменте и смещению потенциалов окисления и восстановления в катодную область. Действительно, для комплексов 1 и 2 , на основе их ИК и ЯМР ${ }^{13} \mathrm{C}$ спектроскопических данных показано, что степень взаимодействия $\mathrm{CO}_{\text {пм }}$..Pt при переходе от $\mathbf{1}$ к $\mathbf{2}$ усиливается и, таким образом, зависит в большей степени от стерического размера лиганда $\mathrm{Ph}_{2} \mathrm{PH}$, чем от его электронодонорной способности [25].

Влияние природы лигандов в комплексах 1-6 обуславливает и различие в схемах их редокс-превращений. Так, близость значений потенциалов второй (комплекс 4) или третьей волны окисления биядерных комплексов 1, 2 и 5 с Е Е волны окисления моноядерного винилиденового комплекса марганца 7 (табл., рис. 1) свидетельствует о том, что одноэлектронное окисление комплексов 1, 2 и 4, 5 приводит к разрыву связи Mn-Pt и образованию комплекса 7. Для подтверждения этого предположения проведен электролиз при контролируемом потенциале предельного тока первой волны окисления комплексов 1,2 и 4,5 , а также их химическое окисление с использованием $\left[\mathrm{Fc}_{0}\right]\left[\mathrm{BF}_{4}\right]$, с последующей идентификацией продуктов окисления комплексов методами электрохимии и ИК-спектроскопии. Действительно, в ИКспектрах растворов, отобранных как из электрохимической ячейки после ЭКП, так и после химического окисления комплексов, в области валентных колебаний СО групп наблюдаются две полосы с частотами (1994 и 1920 см$^{-1}$ ), соответствующими колебаниям карбонильных групп комплекса 7.

Таким образом, установлено, что одноэлектронное окисление биядерных комплексов 1, 2 и $\mathbf{4}, \mathbf{5}$ на Pt и СУ электродах приводит к отщеплению платиносодержащих фрагментов и образованию моноядерного фенилвинилиденового комплекса марганца 7. На основании полученных результатов предложена следующая схема окисления изученных комплексов:

$$
\begin{aligned}
& \mathrm{Cp}(\mathrm{CO})_{2} \mathrm{MnPt}(\mu-\mathrm{C}=\mathrm{CHPh})(\mathrm{L})\left(\mathrm{L}^{\prime}\right) \stackrel{-\mathrm{e}^{-}}{\longrightarrow}\left[\mathrm{Cp}(\mathrm{CO})_{2} \mathrm{MnPt}(\mu-\mathrm{C}=\mathrm{CHPh})(\mathrm{L})\left(\mathrm{L}^{\prime}\right)\right]^{+} \rightarrow \\
& \rightarrow \mathrm{Cp}(\mathrm{CO})_{2} \mathrm{Mn}=\mathrm{C}=\mathrm{CHPh}+\left[\mathrm{Pt}(\mathrm{L})\left(\mathrm{L}^{\prime}\right)\right]^{+\cdot} \text { solv } \\
& \mathrm{Cp}(\mathrm{CO})_{2} \mathrm{Mn}=\mathrm{C}=\mathrm{CHPh} \stackrel{-\mathrm{e}^{-}}{\longrightarrow}\left[\mathrm{Cp}(\mathrm{CO})_{2} \mathrm{Mn}=\mathrm{C}=\mathrm{CHPh}^{+} .\right.
\end{aligned}
$$

На вольтамперограммах биядерных трикарбонильных комплексов 3 и 6 на Pt- и СУэлектродах после их одноэлектронного окисления в отличие от комплексов 1, 2 и 4, 5 отсутствует волна, соответствующая окислению моноядерного комплекса 7 (табл.). При этом одно-

$$
-548-
$$


электронное окисление комплексов 3 и 6 протекает квазиобратимо (отношение предельных токов катодного и анодного пиков первой стадии окисления комплекса $3 \mathrm{I}_{\mathrm{pc}} / \mathrm{I}_{\mathrm{pa}}=0.6$ (рис. $1 a$, пики $\mathrm{A}_{1}, \mathrm{~A}_{1}$ ), что свидетельствует о большей устойчивости комплексов 3 и 6 в редокс-реакциях по сравнению с комплексами 1, 2 и 4, 5.

Следовательно, в отличие от электрохимического одноэлектронного окисления соединений 1,2 и 4, 5, комплексы 3 и 6 окисляются квазиобратимо по следующей схеме:

$$
\mathrm{Cp}(\mathrm{CO})_{2} \operatorname{MnPt}(\mu-\mathrm{C}=\mathrm{CHPh})(\mathrm{L})(\mathrm{CO}) \underset{+\mathrm{e}^{-}}{\stackrel{-\mathrm{e}^{-}}{\rightleftarrows}}\left[\mathrm{Cp}(\mathrm{CO})_{2} \operatorname{MnPt}(\mu-\mathrm{C}=\mathrm{CHPh})(\mathrm{L})(\mathrm{CO})\right]^{+} .
$$

Полученные электрохимические данные подтверждаются результатами квантовохимического исследования «родственных» $\mu$-винилиденовых комплексов $\mathrm{Cp}(\mathrm{CO})(\mathrm{L}) \mathrm{MnFe}(\mu-$ $\mathrm{C}=\mathrm{CHPh})(\mathrm{CO})_{4}\left(\mathrm{~L}=\mathrm{PPh}_{3}, \mathrm{PPh}_{2} \mathrm{H}, \mathrm{PPhH}_{2}, \mathrm{PH}_{3}, \mathrm{CO}\right)$ [29], согласно которым основной причиной уменьшения стабильности комплексов в ряду $\mathrm{L}=\mathrm{CO}>\mathrm{PH}_{3}>\mathrm{PPhH}_{2}>\mathrm{PPh}_{2} \mathrm{H}>\mathrm{PPh}_{3}$ является постепенно увеличивающийся размер конического угла фосфинового лиганда (L) при атоме марганца.

\section{Заключение}

Электрохимическое изучение гетеробиядерных комплексов $\mathrm{Cp}(\mathrm{CO})_{2} \operatorname{MnPt}(\mu-\mathrm{C}=\mathrm{CHPh})(\mathrm{L})$ $\left(\mathrm{L}^{\prime}\right)(\mathbf{1 - 6})$ показало, что их редокс-свойства (значения потенциалов окисления и восстановления, схемы их редокс-превращений, стабильность в редокс-реакциях) зависят от природы лигандов L, L' при атоме платины. При этом решающим фактором являются структурные изменения марганец-платиновых комплексов, происходящие в результате введения различных фосфорсодержащих лигандов в координационную сферу атома платины, что приводит к разной степени полумостикового взаимодействия между атомом платины и соседней карбонильной группой атома марганца.

\section{Список литературы}

1. Ungvary F. Application of transition metals in hydroformylation. Annual survey covering the year 2000. Coord. Chem. Rev. 2001. Vol. 218, P. 1-41.

2. Labinger J.A. Platinum-catalyzed $\mathrm{C}-\mathrm{H}$ functionalization. Chem. Rev. 2017. Vol. 117, P. 8483-8496.

3. Belluco U., Bertani R., Michelin R.A., Mozzon M. Platinum-alkynyl and-alkyne complexes: old systems with new chemical and physical perspectives. J. Organomet. Chem. 2000. Vol. 600, P. 37-55.

4. Wang T., Keyes L., Patrick B.O., Love J.A. Exploration of the mechanism of platinum(II)catalyzed C-F activation: Characterization and reactivity of platinum(IV) fluoroaryl complexes relevant to catalysis. Organometallics 2012. Vol. 31, P. 1397-1407.

5. Wang T., Keyes L., Patrick B.O., Love J.A. Exploration of the mechanism of platinum(II)catalyzed C-F activation: Characterization and reactivity of platinum(IV) fluoroaryl complexes relevant to catalysis. Organometallics 2012. Vol. 31, P. 1397-1407.

6. Rosenberg, Laine R. Concepts and models for characterizing homogeneous reactions catalyzed by transition metal cluster complexes. In catalysis by di- and polynuclear metal cluster complexes Eds. Adams R.D., Cotton F.A. Wiley-VCH: New York, 1998. Ch. 1, P. 1.

$$
-549-
$$


7. Adams R.D. Metal segregation in bimetallic clusters and its possible role in synergism and bifunctional catalysis. J. Organomet. Chem. 2000. Vol. 600(1-2), P. 1-6.

8. Adams R.D., Captan B. Bimetallic cluster complexes: synthesis, structures and applications to catalysis. J. Organomet. Chem. 2004. Vol. 689(24), P. 4521-4529.

9. Buchwalter P., Rosé J., Braunstein P. Multimetallic Catalysis Based on Heterometallic Complexes and Clusters. Chem. Rev. 2015. Vol. 115, P. 28-126.

10. Antonova A.B. Use of the $\mathrm{Mn}=\mathrm{C}=\mathrm{C}$ system in organometallic and organic synthesis. Coord. Chem. Rev. 2007. Vol. 251, P. 1521-1560.

11. Bruneau C., Dixneuf P.H. Metal Vinylidenes in Catalysis. Acc. Chem. Res. 1999. Vol. 32, P. 311-323.

12. Bruce M.I. Organometallic chemistry of vinylidene and related unsaturated carbenes. Chem. Rev. 1991. Vol. 91, P. 197-257.

13. Jutand A. Contribution of electrochemistry to organometallic catalysis. Chem. Rev. 2008. Vol. 108, P. 2300-2347.

14. Zanello P., Fabrizi de Biani. F. Bimetallic effects on the redox activity of transition-metal carbonyl clusters. In Metal clusters in chemistry. Eds. Braunstein P., Oro L.A., Raithby P.R. WileyVCH: New York, 1999. V. 2, P. 1104-1136.

15. Bruneau C., Dixneuf P.H. Metal Vinylidenes in Catalysis. Acc. Chem. Res. 1999. Vol. 32, P. 311-323.

16. Antonova A.B., Kovalenko S.V., Korniyets E.D., Petrovsky P.V., Gulbis G.R., Johansson A.A. Chemistry of vinylidene complexes. III. Binuclear manganese-platinum complexes with bridging phenylvinylidene ligand. Inorg. Chim. Acta. 1985. Vol. 96, P.1-7.

17. Antonova A.B., Kovalenko S.V., Korniyets E.D., Petrovsky P.V., Johansson A.A., Deykhina N.A. Chemistry of vinylidene complexes. V. The ligand substitution reactions at the platinum atom in complexes $\mathrm{Cp}(\mathrm{CO})_{2} \mathrm{MnPt}(\mu-\mathrm{C}=\mathrm{CHPh}) \mathrm{L}_{2}$. Inorg. Chim. Acta 1985. Vol. 105(2), P. 153-163.

18. Чудин О.С., Верпекин В.В., Кондрасенко А.А., Павленко Н.И., Рубайло А.И. Синтез и физико-химические свойства фенилвинилиденовых комплексов с остовом $\mathrm{MnPt}$, содержащих дифенилфосфиновый лиганд при атоме платины. Журн. СФУ. Химия 2018. Т. 11(3), С. 390-400. [Chudin O.S., Verpekin V.V., Kondrasenko A.A., Pavlenko N.I., Rubaylo A.I. Syntheses and physicalchemical properties of phenylvinylidene complexes with MnPt core, containing diphenylphosphine ligand at the platinum atom. Russian Journal of Siberian Federal University. Chemistry 2018. Vol. 11(3), C. 390-400 (In Russ)].

19. Вайсбергер Ф., Проскауэр Э., Диддик Дж., Тупс Э. Органические растворители. Физические свойства и методы очистки. М.: Иностр. лит., 1958. 519 с. [Weisberger F., Proskauer E., Diddik J., Toops E. Organic solvents. Physical properties and purification methods. Moscow: Foreign. Lit., 1958. 519 p. (In Russ.)]

20. Connelly N.G., Geiger W.E. Chemical redox agents for organometallic chemistry. Chem. Rev. 1996. Vol. 96, P. 877-910.

21. Иогансон А.А., Антонова А.Б., Трухачева В.А., Бурмакина Г.В., Рубайло А.И., Максимов Н.Г., Коваленко С.В., Дейхина Н.А. Химия винилиденовых комплексов. Сообщ. 6. Электрохимическое восстановление и УФ-спектры винилиденовых производных цимантрена и комплексов со связью Mn-Pt. Изв. АН СССР. Сер. хим. 1987. № 1, С. 56-62. [Ioganson A.A., 
Antonova A.B., Trukhacheva V.A., Burmakina G.V., Rubaylo A.I., Maksimov N.G., Kovalenko S.V., Deykhina N.A. Chemistry of vinylidene complexes. VI. Electrochemical reduction and UV spectra of vinylidene derivatives of cymantrene and complexes with the Mn-Pt bond. Izv. Akad. Nauk SSSR, Ser. Khim. 1987. No. 1, P. 56-62. (In Russ.)]

22. Бурмакина Г.В., Павленко Н.И., Иогансон А.А., Антонова А.Б., Лукичева О.В., Рубайло А.И., Новикова Т.П., Погребняков Д.А. Электрохимическое восстановление фенилвинилиденовых комплексов, содержащих Mn, Fe и Pt. Журн. общей химии. 2000. T. 70(12), С. 20292035. [Burmakina G.V., Pavlenko N.I., Ioganson A.A., Antonova A.B., Lukicheva O.V. Rubaylo A.I. Novikova T.P., Pogrebnykov D.A. Electrochemical reduction of phenylvinylidene complexes containing Mn, Fe and Pt. Russian Journal of General Chemistry 2000. Vol. 70(12), P. 2029-2035. (In Russ.)]

23. Antonova A.B., Kovalenko S.V., Korniyets E.D., Petrovsky P.V., Gulbis G.R., Johansson A.A. Chemistry of vinylidene complexes. III. Binuclear manganese-platinum complexes with bridging phenylvinylidene ligand. Inorg. Chim. Acta. 1985. Vol. 96, P. 1-7.

24. Antonova A.B., Kovalenko S.V., Korniyets E.D., Petrovsky P.V., Johansson A.A., Deykhina N.A. Chemistry of vinylidene complexes. V. The ligand substitution reactions at the platinum atom in complexes $\mathrm{Cp}(\mathrm{CO}) 2 \mathrm{MnPt}(\mu-\mathrm{C}=\mathrm{CHPh}) \mathrm{L} 2$. Inorg. Chim. Acta 1985. Vol. 105(2), P. 153-163.

25. Чудин О.С., Верпекин В.В., Кондрасенко А.А., Павленко Н.И., Рубайло А.И. Синтез и физико-химические свойства фенилвинилиденовых комплексов с остовом $\mathrm{MnPt}$, содержащих дифенилфосфиновый лиганд при атоме платины. Журн. СФУ. Химия 2018. Т. 11(3), С. 390-400. [Chudin O.S., Verpekin V.V., Kondrasenko A.A., Pavlenko N.I., Rubaylo A.I. Syntheses and physicalchemical properties of phenylvinylidene complexes with $\mathrm{MnPt}$ core, containing diphenylphosphine ligand at the platinum atom. Russian Journal of Siberian Federal University. Chemistry 2018. Vol. 11(3), C. 390-400 (In Russ)].

26. Вайсбергер Ф., Проскауэр Э., Диддик Дж., Тупс Э. Органические растворители. Физические свойства и методы очистки. М.: Иностр. лит., 1958. 519 с. [Weisberger F., Proskauer E., Diddik J., Toops E. Organic solvents. Physical properties and purification methods. Moscow: Foreign. Lit., 1958. 519 p. (In Russ.)]

27. Connelly N.G., Geiger W.E. Chemical redox agents for organometallic chemistry. Chem. Rev. 1996. Vol. 96, P. 877-910.

28. Tolman C.A. Steric effects of phosphorus ligands in organometallic chemistry and homogeneus catalysis. Chem. Rev. 1977. Vol. 77(3), P. 313-348.

29. Иванова-Шор Е.А., Шор А.М., Наслузов В.А., Рубайло А.И. Квантово-химическое исследование влияния фосфинового лиганда на структуру винилиденового биядерного комплекса Mn и Fe. ЖСX 2016. Т. 57(2), С. 283-291. [Ivanova-Shor E.A., Shor A.M., Nasluzov V.A., Rubailo A.I. A quantum chemical study of the effect of phosphine ligand on the structure of the Mn and Fe vinylidene binuclear complex. Journal of Structural Chemistry 2016. Vol. 57(2), P. 267-274. (In Russ.)]

30. Johansson A.A., Antonova A.B., Pavlenko N.I., Rubailo A.I. Infrared study of transformation of a terminal carbonyl ligand into a bridging one in the MnPt and MnPd $\mu$-vinylidene complexes. J. Mol. Structure 1997. Vol. 408/409, P. 329-332. 